and one of laryngismus. Four cases were reported to the manufacturer (Beecham Research Laboratories, personal communication) one patient had severe dyspnoea, extension of the neck, and a sensation of the tongue being forced back; and another had protrusion of the tongue and upper airway obstruction. Two men with stridor, one with anxiety and one with dysphagia and dyspnoea, were reported to the Australian Drug Evaluation Committee (personal communication). A swollen tongue occurred in 20 of 116 dystonic reactions in 1152 psychiatric inpatients. ${ }^{2}$ These reports were not detailed, and the reactions may not have been dystonic.

Doctors should be aware that neuroleptic drugs may cause alarming upper airway obstruction with atypical clinical features and should be prepared to administer benztropine mesylate when this occurs.

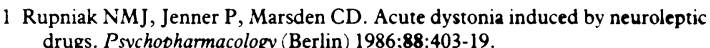

(Accepted 19 fuly 1988)

\section{Surveillance of AIDS cases: How acceptable are the figures?}

\author{
Celia Duff, J Peter Hutchby
}

Department of Community Medicine, East Anglian Regional Health Authority, Cambridge CB4 1RF

Celia Duff, BM, senior registrar

J Peter Hutchby, FFCM, regional specialist

Correspondence to: Dr Celia Duff, Huntingdon Health Authority, Huntingdon PE18 6SE.

The AIDS (Control) Act 1987 requires health authorities and health boards to report yearly the number of AIDS cases known to them. Figures supplied by the Communicable Disease Surveillance Centre are recommended for the purpose. National surveillance of AIDS was established in $1982^{1}$ and is partly based on voluntary confidential reports from clinicians. An objective of the surveillance is to provide information for health care planning.

The number of cases ascribed nationally to the East Anglian region was below that noted by one local district, and a retrospective regional study was initiated during July 1987 to resolve the discrepancy. We report on the results of the study and of regionally coordinated surveillance subsequently developed to increase the accuracy of monitoring.

\section{Methods and results}

A specialist in community medicine in each district ascertained confidentially the details of all people with AIDS known to the health care services. The details included name, sex, date of birth, and current district of residence or date of death. Checks were also made of the data of the regional Hospital Activity Analysis and of the death register from 1982 against the International Classification of Diseases rubric 279.1 (deficiency of cell mediated immunity) then used for AIDS. Nineteen possible cases were identified.

Reporting of 19 possible cases of AIDS in East Anglia to Communicable Disease Surveillance Centre, Fuly 1987

\begin{tabular}{lc}
\hline & No of cases \\
\hline AIDS reported by region & 6 \\
AIDS not reported & 7 \\
AIDS reported elsewhere` and treated in region & 1 \\
Non-AIDS, HIV positive & 3 \\
Non-AIDS, immune deficiency (correctly coded) † & 2 \\
\hline *North West Thames. & \\
†According to International Classification of Diseases code 279.1.
\end{tabular}

One of us visited each district to verify the diagnosis after discussion with clinicians and examination of the case notes. The full list of verified cases, with names coded according to the Soundex protocol, ${ }^{2}$ was sent to the Communicable Disease Surveillance Centre for cross checking. Analysis showed that the correct cumulative total of cases first reported from East Anglia to the end of June 1987 should have been 13 (table).

A regionally coordinated surveillance scheme started from August 1987. Confidential links were established, through district community physicians, with all clinicians concerned with care of people with AIDS. Information was passed to the regional coordinator, who also has access to computerised data from inpatient, death, and cancer registers. Relevant disease codes were listed quarterly and clinical details examined to confirm or exclude AIDS. The information was exchanged with the Communicable Disease Surveillance Centre and details supplied of cases reported in other regions when the patients were residing in East Anglia. The regional scheme collated cases in three ways: by district of reporting, district of residence, and district of treatment.

\section{Comment}

Our retrospective study showed the potential for confusion in collating AIDS cases. Nationally cases were counted by district or region of reporting whereas experience in East Anglia showed that the method did not usefully indicate the use of and the possible need for health services locally. People with AIDS may initially seek diagnosis in one district or region while resident in another, from which they may subsequently receive hospital and community care.

Epidemiological surveillance contributes to service planning by enhancing the accuracy of predictions of service requirements. Because of the three methods of collating data in the newly developed regional surveillance the districts had a fuller picture of the impact of the epidemic locally, and predictions have been made with the data of the need for hospital and community services at district, county, and regional levels. ${ }^{3}$ The scheme has proved both acceptable and useful. The increased accuracy and timeliness of reporting is largely due to the local lines of communication with a facility for personal contact. Had this exercise not taken place we believe that the seven unreported cases in July 1987 would have remained unreported. The clinicians concerned were grateful for reassurance regarding confidentiality and clarification of reporting procedures.

Other regions might consider adopting this approach to provide locally useful data while complying with the AIDS (Control) Act.

We thank Rhys Williams of the Cambridge University department of community medicine, Andrew Tannahill of the East Anglian Regional Health Authority, our clinical colleagues, and the PHLS Communicable Disease Surveillance Centre, for their help and cooperation.

1 Communicable Disease Surveillance Centre. Surveillance of the acquired immune deficiency syndrome in the United Kingdom, January 1982-July 1983. Br Med f 1983;287:407-8.

2 Fenna D. Phonetic reduction of names. Computer. Programs in Biomedicin 1984;19:31-6.

Tennison BR, Hagard S. AIDS: predicting cases nationally and locally. Br Med $\mathcal{Y}$ 1988;297:711-3.

(Accepted 14 September 1988) 